\title{
SpeakUp - A Mobile App Facilitating Audience Interaction
}

\author{
Adrian Holzer ${ }^{1}$, Sten Govaerts ${ }^{1}$, Jan Ondrus ${ }^{2}$, Andrii Vozniuk ${ }^{1}$, David Rigaud ${ }^{3}$, \\ Benoît Garbinato $^{3}$, and Denis Gillet ${ }^{1}$ \\ 1 EPFL, 1015 Lausanne, Switzerland \\ 2 ESSEC Business School, 95021 Cergy, France \\ 3 University of Lausanne, 1015 Lausanne, Switzerland \\ adrian.holzerdepfl.ch, sten.govaerts@epfl.ch, ondrusdessec.edu, \\ andrii.vozniukeepfl.ch, ddrigaudegmail.com, \\ denis.gilleteepfl.ch, benoit.garbinato@unil.ch
}

\begin{abstract}
A dynamic student-teacher interaction during class is an important part of the learning experience. However, in regular class settings and especially in large classrooms, it is a challenging task to encourage students to participate as they tend to be intimidated by the size of the audience. In this paper, in order to overcome this issue, we present SpeakUp, a novel context-aware mobile application supporting the social interactions between speakers and audiences through anonymous messaging and a peer rating mechanism. Context-awarness is achieved by bounding interactions in space and time using location-based authorization and message boards with limited lifetime. Anonymity is used as an icebreaker, so students dare writing down any question that pops in their heads. Peer rating is used to make it easy for teachers to access the most relevant ones and address them. We performed an evaluation with 140 students over five fourhour lessons that indicate that SpeakUp is easy to use and is perceived as useful.
\end{abstract}

Keywords: temporary social media, context-aware mobile interactions, backchannels

\section{Introduction}

Promoting interactivity in conferences and lectures remains challenging, although it is an important success factor in classroom learning [5]. Over the last decade, the use of so-called clickers has become more prevalent in classrooms to address this issue. Clickers are special devices that look like remote controls. Clickers allow students to anonymously answer questions and afterwards teachers can access the aggregated answers. Clickers and other classroom interaction systems are generally regarded as contributing positively to the learning process $[18,2,16,4]$.

Most of the existing systems require dedicated hardware and are typically based on a master-slaves interaction mode, meaning that only teachers can initiate interactions. With mobile smartphones hitting a very high penetration rate and the recent emergence of mobile apps and social media, it has become interesting to leverage mobile devices to design more advanced tools enabling a low setup cost to support interactions in audiences (e.g. in a conference or classroom). 
Some systems, such as MARS [10] or TurningPoint ${ }^{4}$, have simply moved clickerbased interactions (such as multiple choice questions) on mobile phones. But with the communication services offered by these devices, it is also possible to interact more indirectly with students by tapping into secondary communication channels, sometimes refered to as backchannels.

For example, students may casually communicate using a mainstream social media platform during class. One way for speakers to get access to this interaction data is to direct backchanneling to a single defined media. This is what many conferences do when they advise their audience to post their comments and questions to generic social media, such as Twitter. Typically, the audience tweets questions using a predefined hashtag (e.g. \#icw12013) so they can be picked out by the conference attendees and organisers.

We believe that the recent widespread penetration of smartphones offers many exciting possibilities for construction of efficient backchannels in classroom environments.

With this in mind, in the next section we present the scope of our mobile application, SpeakUp. Afterwards in Section 3 we elaborate on the related work and then we present the requirement analysis in Section 4. In Section 5 we briefly explain the design and implementation of SpeakUp. Following this, we discuss open research questions in Section 7 and wrap up with a conclusion and future work in Section 8.

\section{Contribution}

SpeakUp is a novel context-aware mobile application specifically designed to support the interactions between speakers and their audiences in both educational and more general settings. At its essence SpeakUp can be seen as a shared message board for the audience of a talk with the following four specific features:

- Location-based authorisation. SpeakUp targets an audience located together in the same physical room by restricting access to a virtual chat room based on location.

- Absence of login barrier. SpeakUp aims at encouraging participation by allowing anonymous posting with no registration and login overhead.

- Peer reviews. SpeakUp filters out irrelevant inputs, by providing a peer-reviewed rating system and relevance-based sorting.

- Here and now conversations. Conversations in SpeakUp keep some of the properties of real-world conversations with an audience. Since messages in SpeakUp are bound in time as well as in space, the sense of privacy increases as only people present here and now can participate in conversations and ratings.

\section{Related work}

As mentioned, the major commercial tools used for supporting audience interactions are clicker-based software, such as TurningPoint or H-ITT ${ }^{5}$ [17] and social media such

\footnotetext{
${ }^{4}$ TurningPoint, www .turningtechnologies .com

${ }^{5}$ H-ITT, http : / /www . h-itt . com
} 
as Twitter ${ }^{6}$. The first two are only designed for two-way interactions between speakers and audiences and generally do not support multi-sided interactions where members of the audience can interact with each other as well as with the speaker. Twitter provides such multi-sided interactions, but not specifically aimed at speaker-audience interactions. They therefore fail to leverage on specific features of these environments, such as physical proximity and the possibility of real-life interactions. There are also more specialized tools in the research literature. We review several of them hereafter and compare them to SpeakUp.

ClassQue. ${ }^{7}$ A tool that is both dedicated to speaker audience interactions (specifically in classrooms) and that also uses social media features [13]. It allows students to ask questions and they can also anonymously comment each other's answer. However, ClassQue is a Java desktop app and there is currently no mobile version. Furthermore it does not offer context-aware facilities.

m-Learning. An early attempt at providing mobile learning support [11] allows student to access the course's content and post questions though the course's wapsite wapsite (i.e. a website using the now extinct WAP technology). There is no rating system for students and it is not clear whether questions are accessible to other students and whether they are anonymous.

TXT-2-LRN. To overcome the burden of dedicated hardware, Scornavacca et al. proposed TXT-2-LRN [15]. This tool allows students to freely ask questions or answer quizzes via SMS directly from their cell phones. The instructor connects her mobile phone to the management tool on her laptop. Students can either send messages on the open channel or answer m-quizes. TXT-2-LRN does not offer any social media features, such as ratings and a message stream.

SHERPA. A recent mobile app targeting students and educators that provides support for social features [14]. This app is used for teacher-student and teacher-teacher interactions. It allows instructors to record attendance and to gather in-class evaluation information. It also allows students to find information about their classmates. SHERPA also enables students and instructors to easily send messages and communicate with each other. However, it does not provide location-based facilities, anonymity, nor support message reviews.

Backchan.nl. A website that aims at collecting all comments and questions of conference attendees [7]. Messages can be rated up or down and the top eight questions are displayed on the presenter's display. Backchan.nl uses pseudonyms as a loose identification mechanism to avoid double voting. From their experience, most users seemed to use their real names and affiliations. However, user names can be changed during a session and thus it is easy to rig the system. In one notable occurance, users coordinated to get eight posts containing the lyrics to "Never gonna give you up" by Rick Astley

\footnotetext{
${ }^{6}$ Twitter, www.twitter.com

${ }^{7}$ ClassQue, http://vip.cs.utsa.edu/classque/
} 
in the top eight, effectively managing to "rickroll" the audience. In backchan.nl rooms are identified by URLs and cannot be discovered by geographical proximity.

\section{Requirement analysis}

For the requirement analysis, we rely on the Value Proposition Canvas [12] as guiding framework, since it provides a simple yet powerful way to think how to establish a good fit between a user's expectations and a tool or service, which could be offered to her (see Figure 1). The canvas has two sides. On the right side, the assumptions about the user are listed. The jobs-to-be-done are the tasks that the user wants to complete. These jobs are associated with pains and gains. Identifying the pains and gains help to have empathy for the user in order to better answer her needs. On the left side, there is the value proposition description. The idea is to list the features of the products and services. More than the specifications, we would like to know how our value proposition can be a gain creator and pain reliever for users.

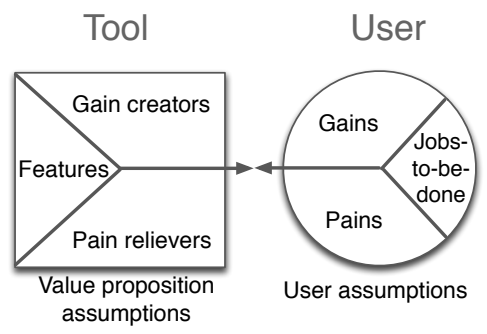

Fig. 1. Value Proposition Canvas [12]

As teachers, students and regular conference attendees, we answered a number of questions related to the different dimensions of the canvas before starting the develoment. The objective was to better understand what jobs students want to solve, what pains they currently have, and what gains they would like to get from a system that aims to improve in-class interactions between teachers and students.

Jobs-to-be-done. For the list of jobs-to-be-done, we found that students may want to be able to participate more actively even when the class is large, exchange ideas in real-time during class, or ask questions whenever they want.

Pains. For the pains, we found that shyness and fear of being ridiculed intimidate many who then fail to engage [8]. Nevertheless, they might have interesting questions, but do not know if others would find them useful or not. Another pain is the amount of irrelevant questions that can be asked. At large conferences, where the Q\&A time is limited, there is a good chance that someone will use up much of the available time elaborating the context of a question that is of no special interest to others. Finally, a general pain associated with using technology in the classroom is the login hurdle that might hinder adoption.

\footnotetext{
${ }^{8}$ Information about rickrolling can be found on wikipedia: http://en.wikipedia.org/ wiki/Rickrolling
} 
Gains. Possible gains include that the audience can learn from the input of others, participation increases the overall interest, and the most relevant questions can be asked.

Features. During the focus group, we also established a number of features that could create pain relievers or gain creators. For instance, the features of SpeakUp are:

- anonymous question sharing and posing

- a transparent login and location-based room discovery

- viewing of others' questions

- rating of questions and relevance-based ordering

Anonymity addresses shyness and encourages participation [6,9]. A transparent login and location-based room discovery reduce usage barriers. Gains are increased by allowing users to view each other's questions. Finally, being able to rate and sort questions allows to filter out irrelevant content and address relevant questions.

\section{The SpeakUp app}

Based on this requirement analysis we devised SpeakUp as shown in Figure 2.

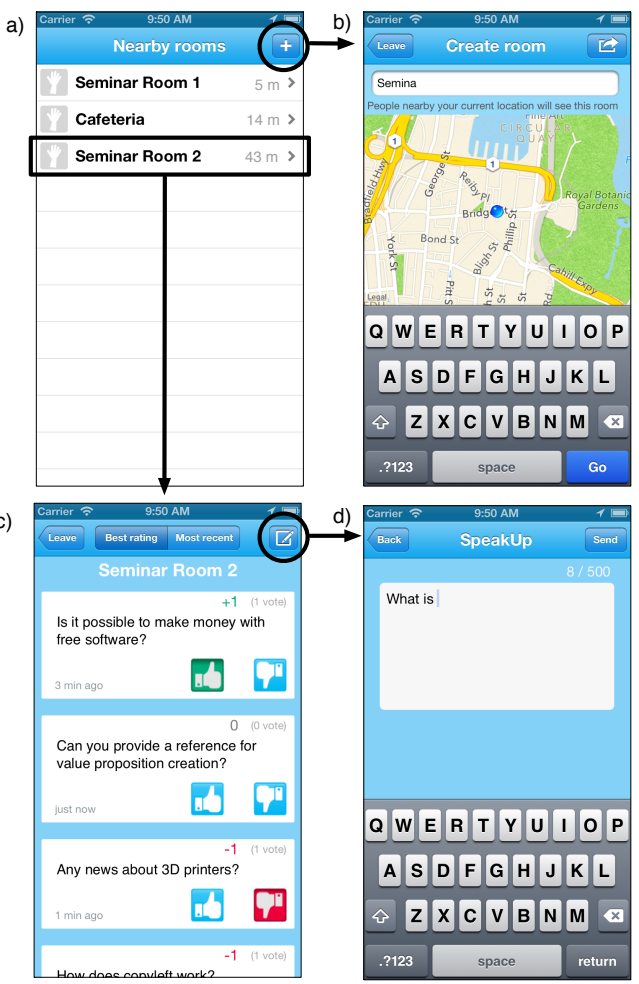

Fig. 2. The SpeakUp app. 
Questions and comments are listed in location-bound chat rooms that function as a virtual metaphor for the physical rooms where the discussions take place. When SpeakUp is launched, the chat rooms in the user's vicinity are displayed (i.e. rooms within a $200 \mathrm{~m}$ radius) as shown in Figure 2-a. Note that the user is never required to enter any personal information in order to use SpeakUp. Figure 2-b shows how users can create their own room at their current location by pressing the plus button on the top right corner. Upon selecting a room, the messages of this room are listed and users can assign a rating to a message by clicking the thumb up or the thumb down icon, as illustrated in Figure 2-c). The messages can be ranked either according to their publication time or to their relevance. The relevance is determined by the rating, i.e. the number of thumbs up minus the number of thumbs down received by a message. Finally, Figure 2-d shows how users can write messages. Note that SpeakUp is implemented for both Apple's iOS and Google's Android. ${ }^{9}$

\section{Evaluation}

We have evaluated SpeakUp in an educational setting over the period of one semester. The evaluation was conducted in a business course on information systems at the University of Lausanne in the fall of 2012 with second year Bachelor students majoring in management and economics. Out of the 350 enrolled students, 140 students between the age of 20 to 25 years, used SpeakUp, as not all students had access to a smartphone. We experimented during the semester with different usage scenarios of SpeakUp. We used SpeakUp in five four-hour sessions. Figure 3 present the results of a voluntary survey we performed after the first session, which inquired about usability, usefulness and the impact on the course (48 out of 140 students completed the survey).

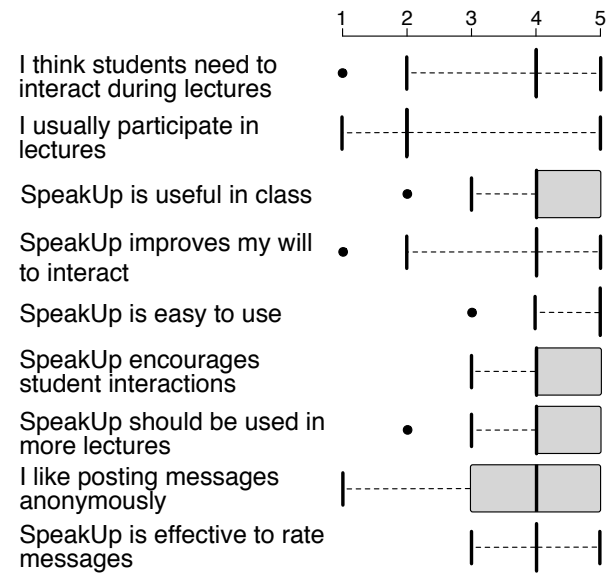

Fig. 3. Boxplot of the likert scale analysis of survey questions (the $\bullet$ 's are outliers).

\footnotetext{
${ }^{9}$ Both versions are freely available at http: //doplab. unil.ch/speakup.
} 
Usability. Some of the survey questions originate from the SUS [1] and PUEU [3] usability surveys but have been recontextualised to SpeakUp. Figure 3 presents the Likert scales ('1 - strongly disagree' to '5 - strongly agree') and their results in a boxplot. A boxplot reads as follows: (1) the vertical lines represent respectively the lowest, the median and the highest value; (2) the outer boundary of the boxes indicate the first and third quartile and (3) the dots correspond to outliers. When the box is missing, the first and third quartiles coincide with the median.

The first two questions were used to further assess our user assumptions from the requirements analysis. That is, whether students thought that part of their jobs-to-bedone was participating in class, and whether they usually engaged in class. The results confirmed these assumptions by showing that most students indeed consider participation as quite an important aspect of a lecture (high median with wide range), but they admit that they usually do not participate (low median with wide range).

Ease of use. The SpeakUp's ease-of-use was rated very high (very high median). Several questions relate to the usefulness of SpeakUp. Students perceived SpeakUp as useful in lectures (high median) and would also like to continue using SpeakUp in other lectures (high median). SpeakUp also seemed to motivate most students to actually interact more (high median, but wide range) and students felt more encouraged to interact when they had access to SpeakUp (high median). From the social media perspective, rating was perceived as an effective means for filtering out irrelevant messages (high median) and most students also preferred the anonymity that SpeakUp provides (high median, but wider range). We did not specifically inquire about the location-boundness of the messages as SpeakUp was always used at the same location.

Ratings. The SpeakUp messages were logged (in total 267 messages and 4354 votes) and three external experts categorised the messages as related to the course organisation, to SpeakUp itself, to the course content or irrelevant (i.e. spam). Figure 4 shows

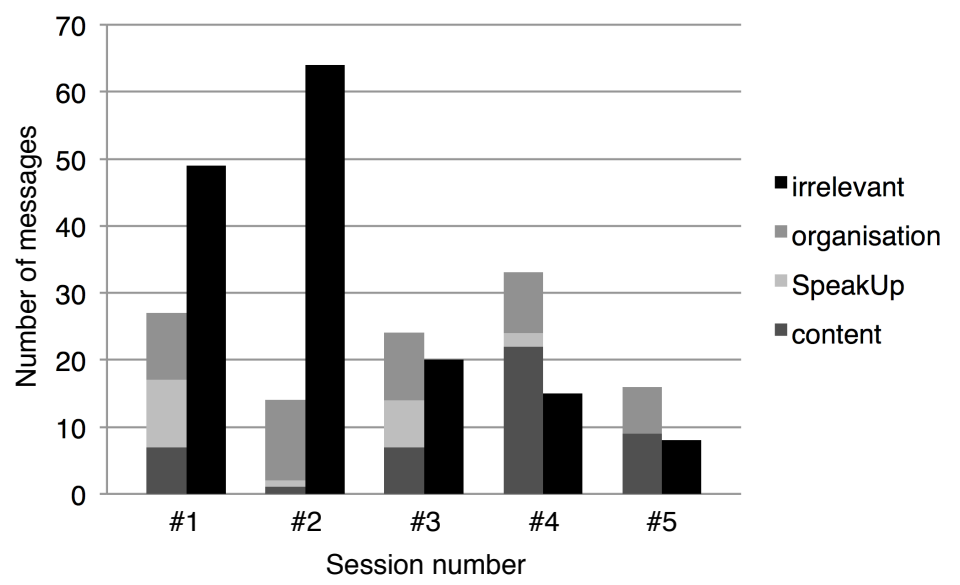

Fig. 4. The categorised number of messages per session. 
this categorisation. The left bar of each session illustrates the relevant messages and their different categories. The right bar summarises the spam messages. In the first session SpeakUp was freely used. During the second session, we moderated the messages, which introduced more spam messages often related to the moderation. In the third lecture, the lecturer discussed the spamming issue with the students. This might have caused the reduced number of irrelevant messages in the last three courses. Figure 4 suggests a novelty factor: in the first sessions SpeakUp is used most, while the total number of messages decreases gradually over the later sessions. Although the total number of messages decreases, the number of relevant messages is more stable. This indicates that SpeakUp is useful for the students. The messages related to the course content are fluctuating, which might be related to the topic of each session.

Figure 5 shows the quantities of positive and negative ratings per category. Overall, it shows that the rating mechanism is effective in discriminating spam. Interestingly, irrelevant messages (e.g. "Please like Christo's page on FB" 45 dislikes, 5 likes, or "Let's all go to the swimming pool" 24 dislikes, 12 likes) get the most ratings and the positive ratings are high. Students seem to try to game the system by upvoting spam, but this is suppressed effectively by the "wisdom of the crowd'. Both Figure 4 and 5 show that organisational messages (e.g. "Can we have the slides before the lecture" 10 dislikes, 65 likes, or "Close the blinds, we can't see anything", 6 dislikes, 43 likes) are very popular and get many positive ratings. The real course content messages are rated less frequently. This might be because organisational questions often affect many students, while content messages might only relate to problems that few experience. Note that the SpeakUp category contains messages that provide feedback on SpeakUp itself (e.g. "I prefer SpeakUp with filter" 9 dislikes, 3 likes, or "To avoid spam, one should be required to log in with the university credentials", 4 dislikes, 9 likes).

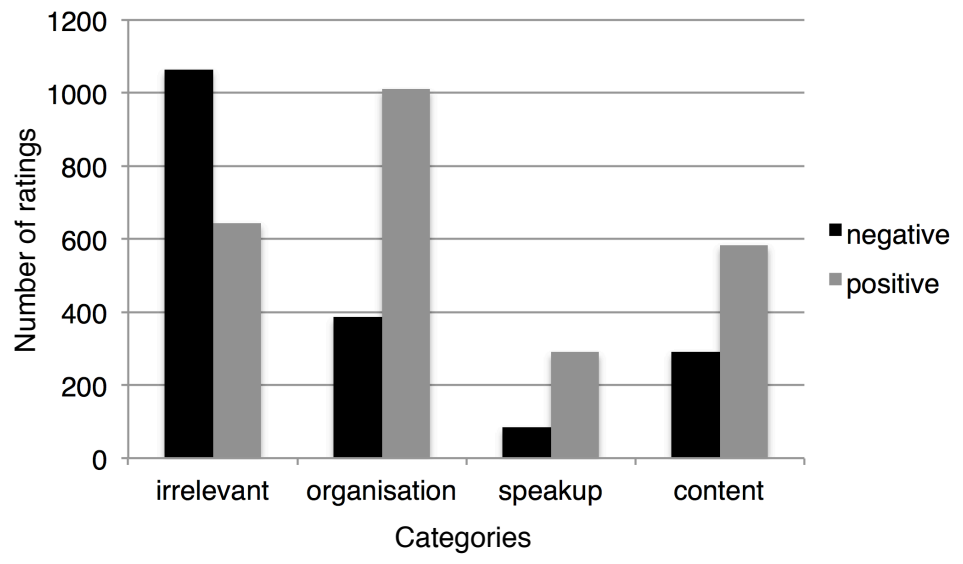

Fig. 5. The positive and negative ratings per category. 
Overview. Overall, we can conclude that SpeakUp seems to be easy to use and useful for the students in this setting. Moreover, SpeakUp motivates most students to participate more in class. The relevant content is filtered out well by SpeakUp's rating mechanism.

\section{Discussion}

Our experience with SpeakUp led to several open issues that are still unresolved by current research and that we believe are worth exploring. In this section, we discuss these issues as open research questions.

How can anonymity be preserved and what is the impact on users? There are certainly situations where users benefit from being anonymous, for instance SpeakUp users are anonymous within a confined group which encourages participation. However anonymity can sometimes lead to spam. The feeling of anonymity within a group can depend on its size (smaller $\rightarrow$ less anonymous). We believe that such parameters of anonymity should be further investigated and might provide a balance between engagement and potential spam.

How do we filter relevant content? Social media can generate information overload and is often targeted by spammers as we witnessed in our evaluation. Therefore, better filtering techniques that sift relevant, novel, interesting and personalised content and that are resilient against spam are needed.

Do social media traces have to exist forever? SpeakUp users can only access messages at a given location for a certain amount of time. ${ }^{10}$ We believe interesting user experiences can be designed with traces limited by time, by location or by action.

Does social validation encourage students to interact directly? After receiving social approval for their questions (i.e. a good positive score), students might find it less intimidating to raise their hand and ask their question directly. We believe it is important to evaluate whether and how SpeakUp really changes the behaviour of students and we plan to evaluate this in the coming months.

\section{Conclusion and future work}

Encouraging live audience interaction is challenging, especially in large classrooms. Existing tools do not fully capture the essence of these interactions, which are inherently local in space and time. To fill this gap, we introduced SpeakUp, a real-time mobile message board that has a strong here and now nature as it restricts access to a virtual space based on physical presence in a certain place at a certain time. This provides an implicit authorisation filter allowing SpeakUp to offer complete anonymity and no login barrier with limited risk of malicious use. Furthermore, it provides support for

\footnotetext{
${ }^{10}$ In SpeakUp, messages are purged after 12 hours.
} 
virtual social interactions as users can see and rate each other's messages, filtering out irrelevant messages.

We evaluated SpeakUp with students and our results convey that SpeakUp is successfully used to address the problem of classroom interaction that they find important. We plan in the next months to deploy SpeakUp in different classrooms and in a conference settings to further evaluate its use. Furthermore, as we pointed out this research raised several unresolved questions that can lead to new exciting research avenues. Finally, this study has focused on student-teacher interactions, future work could expand to investigate how SpeakUp could support interactions among students.

\section{References}

1. Brooke, J. SUS: A quick and dirty usability scale. In Usability evaluation in industry, P. W. Jordan, B. Weerdmeester, A. Thomas, and I. L. Mclelland, Eds. Taylor and Francis, 1996.

2. Caldwell, J. E. Clickers in the large classroom: Current research and best-practice tips. $C B E$ Life Sciences Education 6, 1 (2007), 9-20.

3. Davis, F. D. Perceived usefulness, perceived ease of use, and user acceptance of information technology. MIS Q. 13, 3 (Sept. 1989), 319-340.

4. Elliott, C. Using a personal response system in economics teaching. International Review of Economics Education 1(1) (2003), 80-86.

5. Erickson, J., and Siau, K. e-ducation. CACM 46(9) (2003), 134-140.

6. Freeman, M., Blayney, P., and Glinns, P. Anonymity and in class learning: The case for electronic response systems. AJET 22(4) (2006), 568-580.

7. Harry, D., Gutierrez, D., Green, J., and Donath, J. Backchan.nl: integrating backchannels with physical space. In CHI '08: CHI '08 extended abstracts on Human factors in computing systems, ACM (New York, NY, USA, 2008), 2751-2756.

8. Lantz, M. E. The use of clickers in the classroom: Teaching innovation or merely an amusing novelty? CHB 26, 4 (2010), 556 - 561.

9. Martyn, M. Clickers in the classroom: An active learning approach. EQ 30, 2 (2007), 71-74.

10. McCreath, C., and Leimich, P. Using web-enabled mobile phones for audience participation in database lectures. In In proc. of HEA-ICS Teaching, Learning and Assessment of Databases (TLAD'11) (2011).

11. Motiwalla, L. F. Mobile learning: A framework and evaluation. Comp. \& Education 49, 3 (2007), $581-596$.

12. Osterwalder, A., and Pigneur, Y. Business model generation: A handbook for visionaries, game changers and challengers. Wiley (2011).

13. Robbins, S. Beyond clickers: using classque for multidimensional electronic classroom interaction. In SIGCSE'11 (2011), 661-666.

14. Schweitzer, D., and Teel, S. Sherpa: A mobile application for students and educators in the classroom. In FIE'11 (2011).

15. Scornavacca, E., Huff, S., and Marshall, S. Mobile phones in the classroom: if you can't beat them, join them. CACM 52, 4 (Apr. 2009), 142-146.

16. Sharma, M. D., Khachan, J., Chan, B., and O'Byrne, J. An investigation of the effectiveness of electronic classroom communication systems in large lecture classes. AJET 21 (2005), $137-154$.

17. Siau, K. L., Sheng, H., and Nah, F. F.-H. Use of classroom response system to enhance classroom interactivity. In IEEE Trans. on Education, Vol. 49, No. 3 (2006), pp. 398-403.

18. Yourstone, S. A., Kraye, H. S., and Albaum, G. Classroom questioning with immediate electronic response: Do clickers improve learning? DSJIE 6, 1 (2008), 75-88. 